Linguistik, Poetik, Ästhetik - Abstract

The paper briefly summarizes the proposal made in 1965 that "Poetic Competence" is the basis for creating and evaluating poetry. That this competence lives on, but is different from linguistic competence is exemplified by a close look at the segmental and supra-segmental, morpho-syntactic, lexical, and conceptual structure of Hölderlin's poem "An Zimmern", revealing a surprisingly complex and balanced structure of the apparently simple four lines. The second part of the paper discusses the question whether judgments about poetry are to be studied as based on the relation between specific properties of poems and persons. Finally the problem is raised, whether literary art is based on a specific Poetic Competence, or instantiates a general aesthetic ability applied to linguistic objects. This is construed as a speculative, but still empirical question.

\title{
Manfred Bierwisch
}

\section{Linguistik, Poetik, Ästhetik}

\begin{abstract}
There can hardly be any doubt that poetic ability (in the broad sense) is in part innate and universal, in part acquired, and in part just something about the person that no one knows how to explain

Noam Chomsky
\end{abstract}

\section{Linguistik und Poetik - ein Programm}

Die Neujustierung der Linguistik in den 50er und 60er Jahren des letzten Jahrhunderts machte sich auf unterschiedliche Weise bemerkbar. Zu den Bereichen, in denen unmittelbare Auswirkungen der neuen Ideen am ehesten zu erwarten waren, gehörte die Untersuchung literarischer Texte. Ein programmatisches Exempel dieser Bemühungen war der Band "Mathematik und Dichtung" (1965). Zu diesem Band hatte ich einen Beitrag beigesteuert, der ziemlich großzügig und mit viel Zuversicht die Art beschrieb, in der Vorstellungen aus der Linguistik auf die Analyse und Theorie von Poesie zu übertragen wären, mit dem Blick auf Unterschiede ebenso wie auf Parallelen. Auch wenn heute manches anders zu formulieren wäre, ist der Grundgedanke, der triviale, aber auch interessante Wirkungen erzeugt hat, einer Überprüfung wert.

Literarische Texte generell und poetische im besonderen werden erzeugt und rezipiert auf der Basis von mindestens zwei miteinander zusammenhängenden, aber doch verschiedenen Dispositionen oder Kenntnissystemen, erstens der Beherrschung der jeweiligen Sprache, und zweitens der Kenntnis der besonderen, zusätzlichen Bedingungen, durch die ein Gedicht sich von einer Wegauskunft oder einer Gebrauchsanweisung und eine Novelle von einem Mietvertrag oder einem Wahlprogramm unterscheidet. Bezieht man sich auf die Sprachkenntnis mit G (für "Grammatik" in einem sehr weiten Sinn, sodaß außer den Kombinationsregeln und Strukturprinzipien auch das Wortschatzwissen eingeschlossen ist), dann kann man 
auf den zweiten Kenntniskomplex mit P (für Poetik) Bezug nehmen. So wie die Linguistik in erster Linie, wenn auch nicht nur, zu klären versucht, was $G$ enthält und welche Prinzipien diesem System zugrunde liegen, so müsste sich dann die Theorie der Dichtung mit der Frage befassen, worin P besteht und auf welchen Prinzipien es beruht. Mit einem Programm dieser Art sind mindestens drei Dinge festgelegt.

Erstens: Das, womit sich eine Theorie der Dichtung oder Poesie befasst, sind eigentlich nicht die Eigenschaften von Texten, sondern die Kenntnisstrukturen, aufgrund deren den Texten die besonderen Eigenschaften zukommen, die man etwas künstlich als "Poetizität" zusammenfassen kann. Es geht bei der Analyse von Dichtung also um mentale Strukturen, um die Bedingungen und Ergebnisse interner Verarbeitung. Das ist ebenso selbstverständlich, ja trivial, wie in Wahrheit konsequenzenreich und problematisch.

Zweitens aber lässt sich die Natur von Kenntnisstrukturen, auf denen die Wirkung von Dichtung beruht, nur anhand poetischer Texte und der Reaktionen auf sie erkunden - ganz so, wie man die Kenntnis grammatischer Regeln oder der Bedeutung von Wörtern nur anhand sprachlicher Äußerungen und ihrer Bewertung durch Sprecher der jeweiligen Sprache erkunden und vielleicht auch systematisch erklären kann. Was man betrachtet und nach Möglichkeit systematisiert, sind demnach zwar Eigenschaften poetischer Texte - aber unbedingt im Hinblick auf mentale Strukturen und Prinzipien, auf denen die relevanten Eigenschaften beruhen. Die Mittel und Methoden, die dabei zum Zuge kommen, sind so wenig zu präjudizieren wie die, mit denen die Organisation des Gedächtnisses, die Fähigkeit der Gesichtererkennung oder der Identifizierung der sprachlichen Lautstruktur untersucht wird. Am Beginn steht in all diesen Bereichen die systematische und sorgfältige Analyse intuitiver Feststellungen, aber der Erweiterung des Methodenkanons sind keine dogmatischen Grenzen zu setzen.

Drittens ist damit die keineswegs selbstverständliche Voraussetzung gemacht, daß es mentale Strukturen, die unter P zusammengefaßt werden können, in einer der Sprachkenntnis vergleichbaren Form als Gegenstand rationaler Analyse tatsächlich gibt, daß diese Strukturen und Prinzipien mit kontrollierbaren Verfahren erfasst und erklärt werden können. Bei aller Unterschiedlichkeit ist damit ein der Linguistik vergleichbares Forschungsprogramm umschrieben. Und es schließen sich zwangsläufig weitergehende Fragen an: Hängen die Prinzipien, auf denen $\mathrm{P}$ beruht, mit denen zusammen, die der Musik, dem Tanz, vielleicht auch den verschiedenen Formen bildender Kunst zugrunde liegen? Für die Musik ist ein groß angelegter Ansatz in dieser Richtung von Lerdahl und Jackendoff (1981) formuliert worden.

Während für die Linguistik das Interesse an sprachlichen Kenntnisstrukturen und ihren Prinzipien zu verzweigten und ertragreichen Forschungsprogrammen geführt hat, ist für die Poetik etwas Vergleichbares kaum zu konstatieren. Die Gründe dafür können unterschiedlicher Art sein. Jedenfalls aber wird zu überlegen sein, ob die Frage nach den Prinzipien der poetischen Kompetenz als eines mentalen Systems richtig gestellt ist und in welcher Weise sie zu einem fruchtbaren sinnvollen Forschungsprogramm führen kann. Natürlich sind solche Überlegungen immer und in diesem von Zeitströmungen beeinflussten Gebiet zumal von Positionen, Meinungen und auch Vorurteilen abhängig. Dennoch sollten sie rationaler Argumentation zugänglich sein. Dafür scheint es mir sinnvoll, anstelle abstrakter Erörterungen ohne 
Vorstellung von den tatsächlichen Phänomenen und Möglichkeiten zunächst ein konkretes Exempel zu betrachten.

\section{Ein Beispiel}

\section{1. Das Thema}

Ich will in diesem Sinn ein Beispiel betrachten, das überschaubar, aber unstrittig und exemplarisch den interessierenden Phänomenbereich repräsentiert - ein HöldrlinGedicht, für das nicht strittig ist, ob es als Kunst, auch im emphatischen Sinn, gilt, für das aber zu klären ist, wieso das zutrifft. Vier Bemerkungen dazu vorab.

1. Natürlich werden Eigenschaften des Gedichts zu identifizieren sein. Es geht aber, im Sinn des zu diskutierenden Programms, um die internen Repräsentationen dieser Eigenschaften, und um die Frage, auf welchen Prinzipien sie beruhen. Thema sind also z.B. nicht die phonetischen Eigenschaften, die das Metrum ausmachen und die in der Schriftform nicht einmal vorkommen, sondern die Muster, die sich in ihnen manifestieren, die also dem gedruckten Text nur vermöge der Kenntnis des Lesers zukommen.

2. Bei den dafür nötigen internen Bedingungen darf es nicht um Expertenwissen gehen, sondern nur um das, was ein beliebiger Leser, der den Text versteht, an Voraussetzungen mitbringt. Das heißt einerseits, die entsprechende Sprachkenntnis muß verfügbar sein, aber es soll andererseits kein literaturhistorisches Spezialwissen aufgeboten werden. Ich werde darum im Wesentlichen absehen von den durchaus interessanten, aber eben sekundären Momenten, die die Hölderlin-Philologie zu den biographischen und historischen Bezügen des Gedichts beisteuern kann.

3. Vorausgesetzt werden kann demnach die Kenntnis der Sprache, ihre Charakterisierung ist nicht Teil der Betrachtung. Ich werde deshalb linguistische Strukturen und ihre Darstellung ohne weitere Begründung als gegeben ansehen und ggf. in die Darstellung einbeziehen.

4. Worauf es ankommt, ist gerade das, was die Differenz zu dieser linguistisch zu bestimmenden Struktur ausmacht, was zu ihr hinzukommt oder von ihr abweicht, unabhängig davon in welcher Form ein solcher Überschuß zu repräsentieren ist.

Der Vierzeiler mit der hinzugefügten Widmung [an Zimmern] ist ein einprägsames, bedeutendes und scheinbar sehr einfaches Gedicht, und gewiß eins von den großen im Bereich der Kleinformen der deutschen Literatur.

\section{[ An Zimmern ]}

Die Linien des Lebens sind verschieden, Wie Wege sind und wie der Berge Grenzen.

Was hier wir sind, kann dort ein Gott ergänzen Mit Harmonien und ewigem Lohn und Frieden.

Die Zueignung an den Schreinermeister Zimmer, unter dessen Fürsorge Hölderlin die letzten vier Jahrzehnte seines Lebens verbracht hat, ist kein Titel, sondern eine 
persönliche Geste, die das Gedicht ins scheinbar ganz Private rückt. Daß der Vierzeiler zu den späten Gedichten Hölderlins aus der Phase seiner Krankheit gehört, ist wichtig für die Hölderlin-Philologie. Dem Text selbst ist dieser Umstand nicht (oder allenfalls sehr indirekt) zu entnehmen. Trotz seiner knappen, konzisen Gestalt ist das Gedicht kein Epigramm, seinem Charakter nach ist es keine pointierte Sentenz, sondern so etwas wie ein zusammenfassender Blick auf die Welt.

In ruhiger, fast gelassener Form, die das Versmaß ausdrückt, geht es um Verschiedenheit und Ausgleich, um den Vergleich von Natur und Leben, um Vorläufigkeit und Vollkommenheit, um den Sinn des Ganzen. Dies in etwa nimmt man beim ersten Lesen auf und zugleich die deutliche Zweiteilung des Gedichts in seiner Form: Je zwei Zeilen bilden einen Satz, die Zeilenpaare sind durch das Reimschema $a-b-b-a$ miteinander verschränkt, und sie bilden ganz deutlich ein inhaltliches Gegensatzpaar von Beschreibung und Deutung, von Wirklichkeit und Erwartung und beinahe, aber dann ganz entschieden eben doch nicht von Diesseits und Jenseits.

Dieser intuitive Gesamteindruck wird erzeugt und gestützt durch ein kunstvolles Geflecht von Faktoren, die dem Leser (und übrigens auch dem Autor) keineswegs bewusst sein müssen, so wie dem Sprecher und Hörer einer Aufforderung keineswegs bewusst sein muß, in welcher grammatischen Form sie realisiert wird. Gleichwohl sind die Faktoren im einzelnen zu identifizieren und zusammenzuführen sind. Das will ich in sechs Punkten versuchen.

\section{2. Silbenstruktur und Metrik}

Das Gedicht ist als Gedicht zunächst einfach und eindeutig bestimmt durch Metrik und Versform. Die vier Zeilen sind leicht erkennbar als fünfhebige Jamben, obwohl sich das aus der Schriftform und auch aus der durch sie wiedergegebenen Lautform nicht ohne zusätzliche Kenntnis ergibt. In der üblichen Darstellungsweise haben die Zeilen folgende metrische Form:

$$
u-u-u-u-u-u
$$

Die metrischen Einheiten werden durch Silben konstituiert, die nur sehr ungenau betont " — " und unbetont " $\cup$ " auf die metrische Struktur bezogen werden können. Bereits die vier Silben der ersten Wortgruppe "die Linien" passen gar nicht auf die metrische Gruppe " $\cup-\cup-$ ", die sie bilden müssten, denn die letzte Silbe von "Linien" ist weder lang noch betont. Die tatsächliche Entsprechung zwischen der metrischen und der Silbenstruktur ist für die erste Zeile hier wiedergegeben:

\section{$u-u-u-u-u-u$ \\ Die $\mathrm{Li}$ ni en des Le bens sind ver schieden}

Die keineswegs trivialen Bedingungen der Passfähigkeit, die dem zugrunde liegen, gehören zweifellos zu dem, was die Struktur von P ausmacht. Daß eine tatsächlich unbetonbare Silbe wie die dritte in "Linien" unter genau angebbaren Bedingungen eine schwere Einheit im Metrum bilden kann, entspricht den Regeln von P. Anders ist es mit zwei Unregelmäßigkeiten der letzten Zeile: Wenn "Linien" dreisilbig ist, dann ist "Harmonien" eigentlich viersilbig und hätte damit eine Silbe zu viel. Ebenso hat "ewigem" eine Silbe zu viel, wenn man es nicht zu "ew'gem" kontrahiert. 


\section{$\cup-\cup-(\cup) \cup-(\cup) \cup-\cup-u$ \\ Mit Harmo ni en und ew i gem Lohn und Frieden.}

Die 'überschüssigen' Werte sind eingeklammert, und es ist eine interessante Frage, ob die mit ihnen verbundene Verletzung der regulären Jamben gewollt ist oder ob "Harmonien" und "ewigem" um eine Silbe verkürzt zu realisieren sind, sodaß der regelmäßige Aufbau des Versmaßes gewahrt bleibt ${ }^{1}$. Außer diesem Punkt gibt es in der letzten Zeile aber eine weitere Eigentümlichkeit. Da der Wortakzent von "Harmonien" nicht auf der ersten Silbe liegen und diese nur einen Nebenton erhalten kann, bleibt der erste Versfuß der Zeile ohne Akzent, was nur im Ausnahmefall zulässig ist. Die Realisierung des jambischen Metrums ergibt bei Berücksichtigung der sprachlich vorgegebenen Akzentverhältnisse damit insgesamt ein keineswegs gleichförmiges Muster. Markiert man die Hauptakzentstellen entsprechend, so ergibt sich das folgende (noch immer vereinfachte) Bild von der metrischen Struktur der vier Verszeilen:

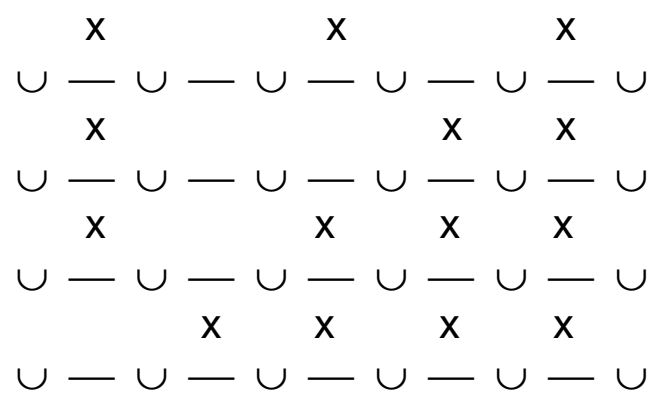

Zweifellos prägt dieser gleichermaßen gebundene wie flexible Rhythmus wesentlich den Grundtenor des Gedichts

\section{3. Reim und Segmentstruktur}

Mit den metrischen Eigenschaften ist die poetische Struktur der Lautform aber natürlich nicht erschöpft. Bereits erwähnt habe ich das offensichtliche und gewissermaßen kanonische Reimschema "verschieden - Frieden, Grenzen ergänzen", das hier zugleich die Zweiteilung des Gedichts bekräftigt und überbrückt. Nicht so offensichtlich, weil in der neueren Poesie weniger systematisch auftretend, ist die bindende Wirkung von Stabreimen, das heißt die segmentale Identität nicht der Coda der Silbe, sondern ihres Anlauts:

Die Linien des Lebens sind verschieden,

Wie Wege sind und wie der Berge Grenzen.

Während Metrik, End- und Stabreim der Lautstruktur gezielte Regularitäten aufprägen, sind weitere Eigenschaften der segmentalen Organisation eher verdeckter, sozusagen untergründiger Natur, aber in einem Gedicht weder zufällig

\footnotetext{
${ }^{1}$ Hier könnte ein Blick in die Hölderlin-Exegese sinnvoll sein. Der ausfahrend freie metrische Gestus etwa in Hölderlins hymnischen Gedichten und sein freier Umgang mit unbetonten Silben könnte ein durchaus beabsichtigtes Aufbrechen der regulären Jamben nahe legen. Andererseits ist nicht nur die resignative Verfassung des kranken Hölderlin, sondern vor allem der ausgleichend ruhige Gestus des vorliegenden Vierzeilers ein Motiv, das eher für die regularisierende Kontraktion spricht, die ich im folgenden annehmen will.
} 
noch ohne Wirkung. Das betrifft in unterschiedlicher Weise die beiden Hauptklassen des segmentalen Inventars.

Für Konsonanten gilt, auch im Deutschen, daß die stimmlosen Obstruenten $\mathrm{p}, \mathrm{t}, \mathrm{k}$ am deutlichsten und zugleich am selbstverständlichsten konsonantenhaft sind. Auffällig ist nun, daß diese drei Segmente, die die neutralste und strengste Form der Verschlussbildung darstellen, ungewöhnlich schwach vertreten sind. Von diesen den drei Obstruenten kommt den insgesamt 44 Silben nur mit einer Ausnahme nur 9 mal der dentale Verschluß und nur am Silbenende vor:

Die Linien des Lebens sint verschieden, Wie Wege sint unt wie der Berge Grenzen. Was hier wir sint, kann dort ein Gott ergänzen Mit Harmonien unt ewigem Lohn unt Frieden.

Klammert man die lediglich der Auslautverhärtung geschuldeten t in "sind" und "und" aus, dann bleiben nur drei mal Instanzen von t und der einzige nicht dentale und nicht auslautende Verschluß in "kann". Auch wenn dies keinem direkten poetischen Muster entspringt, ist die Vermeidung markanter Unterbrechungen durch Verschlusslaute ein bemerkbares Wirkungsmoment in der formalen Struktur, das verstärkt wird durch die entsprechend verstärkte Dominanz stimmhafter, zumal nasaler und liquider und Segmente, von denen I und w überdies durch die erwähnte Stabreimfunktion bekräftigt sind.

Zu vermerken ist schließlich auch eine nicht zufällige Eigenart in der Rolle des Vokalismus. Es ist nicht ganz unproblematisch, ein Grundinventar der Vokale des Deutschen zu identifizieren. Auszugehen ist von mindestens sieben Vokalen, die zudem distinktiv gespannt (lang) und ungespannt (kurz) sein können, sowie von drei Diphthongen. Von diesen Möglichkeiten kommen ö, ü und die Diphthonge au und oi in unserem Gedicht gar nicht vor, im übrigen verteilen sich die Vokale wie folgt (wobei der Doppelpunkt Vokallänge anzeigt und э für das reduzierte e, das Schwa, und $€$ für kurzes offenes e steht):

Die Linien des Lebens sind verschieden, Wie Wege sind und wie der Berge Grenzen. Was hier wir sind, kann dort ein Gott ergänzen Mit Harmonien und ewigem Lohn und Frieden. i: i: i э $€$ e: $\ni$ i $\in \mathrm{i}: \ni$

i: e: э i u i: $€$ e э е э

a i: i: i a $o$ ai $o \in$ e $\ni$

i a o i: u e: э o: u i: э

Eine deutliche Struktur zeigt sich, wenn man berücksichtigt, daß die (technisch gesprochen) nicht-tiefen, nicht-runden Vokale, also i und e und ihre Varianten, eine natürliche Klasse bilden. Diese Klasse besetzt nicht nur 33 der 44 Silben, die jeweils einen Vokal einbringen, sie macht auch fast vollständig den Vokalismus der beiden ersten Zeilen aus und bestärkt von dieser Seite wieder die Zweiteilung des Gedichts: Bis auf ein "und" kommen die 11 Fälle von a, o, u und ai alle in der zweiten Hälfte des Gedichts vor.

Man darf diese gewissermaßen sekundären, emergenten Momente der Lautstruktur nicht überbewerten, aber auch nicht ignorieren. Denn sie sind für den Leser an der Gesamtwirkung sehr wohl beteiligt, und für den Autor wirken sie (wenn auch sicher unwissentlich) an der Textgestaltung mit. 


\section{4. Syntax und Morphologie}

Auf die einfache Grundstruktur, die dadurch entsteht, daß syntaktisch je ein vollständiger Satz genau zwei Verse besetzt, habe ich bereits verwiesen. Die einfache Klarheit der Organisation geht aber noch eine Stufe weiter, denn jeweils die erste Zeile beider Paare bildet den abgeschlossenen Kern des Satzes, dem in der zweiten Zeile eine adverbiale Erweiterung folgt - als Vergleichssatz in der ersten Hälfte, Präpositionalphrase in der zweiten. Und beide Male ist die Fortsetzung in ihrer Struktur als Koordination mit "und" aufgebaut. Diese Transparenz der Struktur wird mit einem Mittel erreicht, das für gesprochene Sprache charakteristisch ist: Ausklammerung oder Extraposition. Ohne diese Option würden die beiden Sätze etwa so heißen:

Die Linien des Lebens sind wie Wege und wie die Grenzen der Berge verschieden. Was wir hier sind, kann dort ein Gott mit Harmonien und ewigem Lohn und Frieden ergänzen

Es ist nicht nur die metrische, sondern auch die inhaltliche Struktur, derentwegen diese Möglichkeit nicht geht: Der formale Aufbau ist in der Form des Gedichts der gedanklichen Architektur viel unmittelbarer angepasst als in dieser grammatischen Ausgangsform.

Im übrigen ist das syntaktische Instrumentarium sparsam, fast minimalistisch. Außer der Kopula, die drei mal in der Form "sind" auftritt und ein viertes Mal in dem eigentlich zweiteiligen Vergleichssatz "wie Wege sind und wie der Berge Grenzen (sind)" mitverstanden werden muß, außer diesem "sind" der Kopulasätze kommt nur die zweiteilige Verbform "kann ergänzen" vor, die der Kern für den zweiten Hauptsatz ist (und phonologisch durch den einzigen anlautenden Verschlusslaut $k$ markiert ist).

Auch im nominalen Bereich wird größte Einfachheit eingehalten: Zwei Genitivkonstruktionen und eine Attribuierung - "die Linien des Lebens", "der Berge Grenzen", und "(mit) ewigem Lohn" -, sonst nur einfache Substantive, keine Komposita, keine Wortbildungen.

Innerhalb dieser Zurückhaltung, die keine Simplizität ist, wird dennoch kompakt organisierte Syntax wirksam: Das direkte Objekt zum Verb "ergänzen" ist ein Relativsatz, der die Ausgangsform "(Das) was wir hier sind" verkürzt und zugleich umstellt zu "Was hier wir sind", sodaß der entscheidende Kontrast von "hier" und "dort" auch metrisch das richtige Gewicht erhält.

Zu den diffizilen Elementen der Syntax gehört das Definitheits-System, das Nominalgruppen konstituiert und vor allem, aber nicht nur, in den Artikelformen greifbar ist. Von den neun Substantiven des Gedichts sind vier artikellos, was im Plural und bei Abstrakta Indefinitheit bedeutet, drei sind mit dem definiten Artikel verbunden und eins mit dem indefiniten Artikel "ein". Die Definitheit hat drei weitere Instanzen: "der Berge Grenzen" verdeckt die grammatisch gegebene Definitheit von "die Grenzen", ferner sind Personalpronomina - also auch "wir" - immer definit, und schließlich ist das bereits erwähnte Objekt "(das) was wir hier sind" definit. Damit ist die 'Referenz-Struktur' des Gedichts syntaktisch folgendermaßen festgelegt (ø gibt den Nullartikel an): 
definit

die Linien, des Lebens, die Grenzen, der Berge wir was hier wir sind

artikellos indefinit $\varnothing$ Wege, ø Harmonien, ø (ewigem) Lohn $\varnothing$ Frieden

indefinit ein Gott

Auf das inhaltliche Korrelat dieser Distinktionen komme ich gleich zurück. Zunächst ist festzuhalten, daß die Lebenslinien und das, was wir "hier" sind, definit ist, während das, was "dort" ergänzt werden kann, Harmonien, ewiger Lohn und Frieden, indefinit ist. Die Vergleiche - "wie Wege und der Berge Grenzen" - verteilen sich zwischen Definitheit und Indefinitheit. Herausgehoben ist der einzig explizit indefinite Artikel in "ein Gott".

\section{5. Lexik}

Die Wörter, die die syntaktische Struktur tragen, sind provisorisch in zwei Gruppen zu ordnen, die ohne theoretischen Anspruch als Inhalts- und Funktionswörter bezeichnet werden können. Die Inhaltswörter - Substantive, Verben, Adjektive bilden erweiterbare Klassen, während die anderen unscharf in einer Gruppe zusammengefassten Einheiten - Artikel, Pronomina, Konjunktionen etc. - ein abgeschlossenes Repertoire bilden. Die 30 Wörter des Gedichts verteilen sich auf diese Klassen folgendermaßen (Mehrfachvorkommen der gleichen Einheit eingeklammert):

Inhaltswörter:

Substantive: Linie, Leben, Weg, Berg, Grenze, Gott, Harmonie, Lohn, Frieden Adjektive: verschieden, ewig

Haupt-Verb: ergänzen

Funktionswörter:

$\begin{array}{llll}\text { Artikel: } & \text { die (der, des), ein } & \text { Pronomen: } & \text { was, wir } \\ \text { Kopula: } & \text { sind (sind, sind) } & \text { Modalverb: } & \text { kann } \\ \text { Konjunktionen: } & \text { wie (wie) und, (und, und) } & \text { Präposition: } & \text { mit } \\ \text { Deiktika: } & \text { hier dort } & & \end{array}$

Die Grenzen sind unscharf und auch nicht entscheidend, denn die Erweiterbarkeit der offenen Klassen wird überhaupt nicht beansprucht. Das Gedicht ruft außer dem relativ jungen Lehnwort "Harmonie" ausschließlich Elemente aus dem festen Grundbestand des deutschen Wortschatzes auf. Und den zentralen Angelpunkt der des Ganzen markiert die inhaltliche Spezifik, nämlich der Kontrast, gerade der Pronominaladverbien "hier" und "dort".

Die mit Anschaulichkeit verbundenen Wörter - "Wege, Grenzen der Berge" rufen ein Landschaftsbild auf, vielleicht Wanderungen, Konturen eines Gebirgszugs, durch die das Gedicht situiert wird, allerdings im Modus des Vergleichs, den das wiederholte "wie" anzeigt und der einen Anhalt gibt für das, was zwar keineswegs abstrakt, aber eben gar nicht anschaulich ist: "die Linien des Lebens", das was sie "verschieden" macht und die Aura des Ausgleichs: "Harmonie, ewiger Lohn, Frieden". 


\section{6. Metaphorik, Analogien}

Die Semantik des Gedichts - und nicht nur der Inhaltswörter - funktioniert in Wahrheit nur aufgrund der immer verfügbaren Analogiebildung, des Vergleichs, durch den Primärerfahrungen zum Erschließen beliebiger Sinnbereiche dienen können. Auch ohne auffällige Metaphorik zu bemühen, wird diese Möglichkeit hier zweifach und in direkt nachzuvollziehenden Schritten wirksam.

Die erste, bereits genannte Analogie macht Wege und Berge mit ihren Konturen zum Bild von unterschiedlicher Lebenserfahrungen. Der Vergleich interpretiert zugleich das Erlebte, "verschieden" verweist auf das Auf und Ab der Wege, aber ohne mehr als eben dies festzulegen. Der Vergleich teilt sich metrisch und syntaktisch genau auf die Verse eins und zwei auf, das erste Paar stellt als Einheit einen ebenso genauen wie zwanglosen Vergleich dar, die eine offene, an der Natur orientierte Sicht auf das Leben im Ganzen anheim stellt.

Der zweite Vergleich, der nur bedingt als Analogie gelten kann, nimmt eben dieses gedeutete Leben, das wir führen - und das "wir" steht ohne Pathos aber doch für die Menschen insgesamt - und stellt es als das "hier" einem "dort" gegenüber, das nun auf eine Weise erschlossen wird, die wirkliche Essenz des Gedichtes ausmacht: Es ist "dort" anders anders als "hier", aber nicht wie in einer Utopie, die die Realität ersetzt, sondern unter einem Horizont, in dem unser Hier-Sein mit Harmonien und Frieden belohnt und ergänzt wird. Der dritte Vers stellt dieses ergänzende Gegenüber her: Die erste Hälfte greift unser (menschliches) Leben auf, die zweite Hälfte führt die Ergänzung ein, und der letzte Vers diese Paares benennt die Aura dieser Ergänzung. Die Schritte dieses Gedankens, der eigentlich kein Vergleich, sondern eine Konstruktion ist, entsprechen genau dem Aufbau der beiden Zeilen: Der Objektsatz "Was hier wir sind" nimmt den Inhalt des ersten Zeilenpaares auf, die Fortsetzung "kann dort ein Gott ergänzen" eröffnet den Horizont der Ergänzung, die Schlusszeile füllt inn aus. Die Syntax und die Metrik dieser Zeilen entsprechen auf atemberaubende Weise dem was in innen vorgeht.

Die dritte Zeile des Quartetts ist die einzige, die syntaktisch und inhaltlich zweigeteilt ist, markiert übrigens durch das lautlich herausfallende $\mathrm{k}$, und sie stellt in dieser Zweiteilung die Spaltung und die Einheit des Gedichts dar. Nun wird deutlich, daß die Linien des Lebens zwar Wege sind und Bergen gleichen, aber Harmonie und Frieden erst als Ergänzung finden. Katastrophen sind nicht angedeutet, aber Mühen und Vergeblichkeit wohl, es brauchte sonst der Ergänzung durch Harmonien, Lohn und Frieden nicht

Die Architektur des Vierzeilers, von der nach dem Gesagten sehr wohl zu sprechen ist, kann sehr vereinfacht so schematisiert werden:

\begin{tabular}{|c|c|c|}
\hline A1 & & $a$ \\
\hline A2 & & $b$ \\
\hline B1.1 & B1.2 & $b$ \\
\hline B2 & & $a$ \\
\hline
\end{tabular}


Das Reimschema ist am Zeilenende angegeben - es ist die offensichtliche, spiegelbildliche Verzahnung der A- und B-Teile. Die 2-er Zeilen sind inhaltlich und syntaktisch die Ausfaltung ihrer Vorgänger. B1 knüpft mit der ersten Hälfte an A an und führt mit der zweiten Hälfte den Vers B2 ein. B1 gibt so die inhaltliche Entsprechung zum Reimschema, und das k von "kann" ist genau der Schnittpunkt dieser Verknüpfung.

\section{7. A-Theologie}

In diesem Panorama gibt es abschließend eine alles übergreifende Dimension, die ich bis jetzt ausgespart habe. Die Verbindung zwischen dem hier und dort entsteht, durch das, was ein Gott ergänzt. Damit ist angezeigt, daß es nicht um den Ausgleich gelegentlichen Missgeschicks geht, sondern um den Sinn des Lebens überhaupt. Gerade darum aber ist die Form, in der dies im Gedicht - in der Schlüsselzeile B1.2 - geschieht, entscheidend.

Der einzige indefinite Artikel des Gedichts steht in der Verbindung "ein Gott", und er hat einen eindeutigen, einen fundamentalen Stellenwert. Wenn es "ein Gott" heißt, dann ist es nicht der Gott der monotheistischen Religion. Als Individuativum wird ein Gott nicht anders als ein Mensch vergleichbar mit anderen. Aber in der monotheistischen Welt ist Gott nicht nur einzig und damit nicht indefinit, sondern definit - so wie "die Sonne", "das Universum" - sondern singulär, personal. "Gott" ist grammatisch und semantisch deshalb kein Individuativum und läßt weder den definiten noch den indefiniten Artikel zu, "Gott" ist ein Eigenname, das Wort nennt den Allmächtigen, es klassifiziert inn nicht. ${ }^{2}$ Man könnte, um diese Überlegung zu neutralisieren, eine poetische Lizenz in Anspruch nehmen: Das Metrum verlangt eine unbetonte Silbe, und in der von der Antike geprägten Vorstellungswelt sind die Götter längst heimisch. Allerdings, eine poetische Lizenz bleibt eine Schwäche, und wenn es ernst wäre, wenn es um ein monotheistisches Bekenntnis ginge, wäre sie durchaus vermeidbar:

Was hier wir sind, das kann Gott dort ergänzen

Diese Formulierung ist möglich, aber sie ist angestrengt, natürlich steht sie dem Original nach, aber nicht nur in der metrischen Gestalt. Das Gedicht ist durch die Vergleiche hindurch eine Weltsicht, und sie ist nicht jenseitig. Beschworen wird nicht der übermächtige Richter des alten Testamentes, "ein Gott" ist eine Metapher in einer nicht-religiöse Welt, sie steht für die Imagination von Korrespondenzen, die den Mangel des Lebens ausgleichen, eine Imagination, an der das Gedicht selbst teilhat.

\section{8. Fazit}

Die Einordnung des Textes in Zusammenhänge von Biographie und Zeitgeschichte kann zweifellos Hintergründe für manche der hier konstatierten Befunde angeben, aber auch Gewinn aus der Strukturanalyse ziehen. Das gelassen Ausgleichende in Haltung und Struktur dieses Textes springt ins Auge, wenn man an das hymnische

\footnotetext{
${ }^{2}$ Natürlich gehören der Plural und die verschiedenen Artikelformen zum sprachlichen Wissen über das Nomen "Gott", weil die Sprachkenntnis nicht einer Religion verpflichtet ist. Aber wenn es um Gott im christlich monotheistischen Verständnis ginge, wäre der Artikel unmöglich, so wie z.B. der Plural von "Mut" unmöglich ist. Ich sehe dabei ganz ab von der Frage, was über die Semantik, also das rein sprachliche Bedeutungswissen für "Gott" zu sagen wäre.
} 
Pathos und das tragisch Verzweifelnde der großen Gedichte Hölderlins mit den entsprechend ausfahrenden Gesten denkt. Zweifellos entspricht diese Verhaltenheit der späten Lebenssituation Hölderlins. Aber man muß nicht mit Taubes (1991) der Meinung sein, Hölderlins Krankheit entspringe aus der Erfahrung der Unmöglichkeit, antiken Mythos und jüdisch-christlichen Monotheismus zu verbinden, um nachzuvollziehen, daß christliche Jenseitserwartung hier keinen Raum hat. Und die seltsame Klarheit, in der die Balance dieser Haltung gelingt, auch wenn sie Intermittenz in der Krankheit ist, trägt ihre Überzeugungskraft in sich selbst. Der Psychiater hat hier keine Zuständigkeit.

All dies ist ergänzend und hilfreich, notwendige Voraussetzung für das Verständnis und die Wertung des Vierzeilers ist es nicht.

Ich denke nicht, daß ich die vier Zeilen mit der extensiven Interpretation überanstrengt habe. Die Analyse ist überdies nicht einmal vollständig, und jede Einzelheit kann durch Sprachkenntnis und Alltagswissen belegt und bestätigt werden. Ich sage nicht, daß jeder Leser bei jedem Lesen oder Hören des Textes alle erwähnten Strukturen und Bezüge realisiert. Aber sie werden wirksam, auch wenn sie nicht bewusst vorgestellt werden, und sie sind zugänglich allein aufgrund von Kenntnis der Sprache und Aufmerksamkeit. Allerdings: Aufmerksamkeit auf was? Das ist die Frage, die mit diesem Beispiel aufgehellt werden sollte. In allen Befunden ging es um einen bestimmten Überschuß oder um zusätzliche Bedingungen über das Primärverständnis des Textes hinaus. Und sicher ist das, was man mit Qualität, Bedeutsamkeit und Schönheit eines Gedichts meint, eng verbunden mit der Vielschichtigkeit und dem Facettenreichtum, die die Analyse sichtbar macht.

Ähnliche Zusatzstrukturen sind allenthalben identifizierbar. Einer der ingeniösesten Vierzeiler des 20. Jahrhunderts ${ }^{3}$ gibt mit einem lexikalisch-grammatischen Minimum - "die einen/ die andern", "im Dunkel/im Licht" "sehen/nicht sehen" - und dem Prinzip von Kontrast und Parallelismus einer Plattitüde Ewigkeitswert:

Denn die einen sind im Dunkel

Und die andern sind im Licht.

Und man siehet die im Lichte

Die im Dunkel sieht man nicht.

Ganz unvollständig ist die Architektur dieses Quartetts, das aus vierhebigen

Trochäen mit dem Reimschema $a-b-c-b$ besteht, wie folgt anzugeben, wobei die Paare 1 und 2 jeweils einen Kontrast und die B-Verse die Parallelismus-Inversion der A-Verse darstellt:

\begin{tabular}{|ll|}
\hline A1 & a \\
\hline A2 & b \\
\hline B2 & c \\
\hline B1 & b \\
\hline
\end{tabular}

Entscheidend ist dabei natürlich die semantische Unterspezifiziertheit, die das Ganze

\footnotetext{
${ }^{3}$ Anders als bei Hölderlins Gedicht handelt es sich hier allerdings nicht um einen eigenständigen Text. Die Genese dieser Strophe ist ein eigenes Kapitel. Sie ist in Fortführung der Moritat von Mackie Messer als Abschluß des Dreigroschenfilms entstanden, gegen den der Autor Brecht aber gerichtlich geklagt hat.
} 
per Analogie und Metaphorik wahlweise zu einer Gesellschaftsdiagnose oder einer Weltdeutung machen.

\section{Empirische Poetik}

\section{1. Eine Alternative?}

Wenn man der Art der Analyse, die im vorigen Abschnitt exemplifiziert werden sollte, im Prinzip folgt und auch die Einzelfeststellungen im Wesentlichen teilt, dann steht dennoch eine verbindliche Vorstellung davon aus, um welche Strukturen - über die hinaus, die die Linguistik zu erklären hat - und welche dafür zuständigen Prinzipien es geht und wie sie zu charakterisieren sind. Für einige Aspekte, insbesondere den Gesamtbereich der Metrik mit den zugehörigen phonologischen Grundlagen und den Parallelismus mit der morphosyntaktischen Basis, gibt es gut begründete und ausformulierte Vorstellungen. Auch sie erfassen freilich nur die Bedingungen für die Struktur von Versen und Parallelismen und nicht ohne weiteres auch deren ästhetische Qualität und Wirkung. Vor allem aber ist offen, wie diese Bedingungen mit anderen Aspekten zusammenwirken, und was in dieses Spektrum insgesamt einzubeziehen ist. Für das, was die sprachliche Struktur einer Äußerung ausmacht, ist dieses Problem im Prinzip klar beantwortet, für die mögliche poetische Struktur der gleichen Ausdrücke ist nicht einmal die Frage hinreichend deutlich.

Welche Faktoren nehmen an der Konstitution poetischer Wirkung teil und wie sind sie zu repräsentieren? Bilden sie einen geschlossenen Gesamtkomplex oder kommen sie von Fall zu Fall zusammen? Sind es überhaupt mehrere Faktoren, oder ist es nur ein Wirkungsmoment?

Ob diese Fragen richtig gestellt sind, ist offen, und sie werden um so ungewisser, je weiter man den intuitiv vorgegebenen Bereich ausdehnt. Wie sind die Analysen und ihre Mittel von Gedichten auf Romane, auf Dramen oder Opern zu übertragen? Was besagt die Analyse von "An Zimmern" für "Hyperion" oder "Faust", oder Becketts "Happy Days", und wie kommt man vom Schlussvers der Moritat von Mackie Messer zur Dreigroschenoper? Gibt es einen gemeinsamen Rahmen, in den auch Bachs "Kunst der Fuge" oder Schönbergs Streichquartette und womöglich auch Leonardos Abendmahl gehören würden?

Ohne alle diese Weiterungen im Auge zu haben, hat Wolfgang Klein (2007) einen Vorschlag entwickelt, wie ästhetische Urteile einer objektiven Untersuchung zu unterziehen sind. Es geht dabei nicht eigentlich um eine Alternative zu dem in Abschnitt 1 rekapitulierten Programm, sondern um eine besondere Art, es zu interpretieren und ihm eine empirische Basis zu geben. Ausgangspunkt ist die oben bereits erläuterte Überlegung, daß es bei der Untersuchung und Erklärung von Poesie nicht um die Eigenschaften der Texte an sich, sondern um deren Wirkung geht. Von Interesse sind also Texteigenschaften relativ zu den Rezipienten. Von dieser Überlegung ausgehend kommt Klein zu folgender Aufgabenstellung:

Man muß bestimmen,

(a) was die relevanten Eigenschaften der Texte sind;

(b) was die relevanten Eigenschaften der Personen sind, auf die sie wirken;

(c) nach welchen Prinzipien diese Eigenschaften miteinander interagieren 
Für (a) kommt im Prinzip die Linguistik auf, wenn man ihr auch die Behandlung von Dingen wie Vagheit, Mehrdeutigkeit sowie Welt- und Kontextwissen überträgt. In die so bestimmten Eigenschaften könnten scheinbar die oben vorgestellten Erläuterungen zu dem Hölderlin-Text eingeordnet werden, allerdings mit einem wesentlichen Zusatz, der wesentliche Konsequenzen für den Stellenwert des Programmpunkts hat: Klein führt zur Erläuterung von (a) Eigenschaften an, die durch Sprachkenntnis bedingt sind und zwar poetische Wirkungen haben können, zu denen aber gerade nicht die poetik-relevanten Zusatzfaktoren wie Metrik, Versbau, Stabreim, Parallelismus oder Kontrastbildung gehören, die den Fokus der oben skizzierten Hölderlin-Analyse bilden, weil sie die poetische Wirkung erklären sollen. Mit anderen Worten, Kleins Punkt (a) zielt auf Eigenschaften, die durch Sprachkenntnis bedingt sind, nicht auf solche, die davon als poetische zu unterscheiden wären. Das bleibt auch gültig für die durchaus aufschlussreichen Beispiele, die Klein seinerseits mit Blick auf Unterschiede in den poetischen Effekten sprachlicher Texte betrachtet.

Diese Feststellung ist entscheidend für die Punkte (b) und (c) des Programms, sie bilden den Kernpunkt des Vorhabens, der programmatisch so zusammengefaßt wird:

\section{"Ästhetische Urteile müssen als Relationen zwischen Eigenschaften von Texten und Eigenschaften von Personen aufgefasst werden. \\ Diese Relationen müssen mit den Methoden untersucht werden, die dem üblichen Vorgehen in den empirischen Wissenschaften entsprechen."}

Während für die Eigenschaften von Texten nach Kleins Überzeugung die Linguistik zuständig ist, sind seine Vorstellungen über die Eigenschaften von Personen, auf die sich empirische Hypothesen über ästhetische Urteile beziehen können, verräterisch vage. Etwas scherzhaft erwägt er, daß es die Haarfarbe wohl nicht sein kann, eher schon das Geschlecht oder das Alter einer Person, am ehesten aber so etwas wie Vertrautheit mit literarischen Texten im Allgemeinen und dem jeweils zu beurteilenden im Besonderen ${ }^{4}$. Was diese Überlegungen zeigen, ist allerdings klar: Eigenschaften einer Person sind für ihr ästhetisches Urteil gerade so weit von Belang, wie sie die Fähigkeit zur Rezeption des jeweiligen Textes betreffen - und zwar nicht hinsichtlich seiner historischen Wahrheit oder sachlichen Nützlichkeit, sondern genau seiner poetischen Qualität. Das aber heißt nichts anderes, als daß die Eigenschaften, um die es bei der Erklärung ästhetischer Urteile geht, eben die Fähigkeit ausmachen, die spezifisch poetischen Eigenschaften eines Textes zu erfassen und zu bewerten.

Die These, daß poetische Urteile als Relationen zwischen Eigenschaften von Texten und Eigenschaften von Personen aufzufassen sind, erweist sich insofern als reine Formulierungsvariante der Konzeption aus Abschnitt 1: Statt um Relationen zwischen Eigenschaften von Texten und Eigenschaften von Personen geht es tatsächlich um

\footnotetext{
${ }^{4}$ Klein meint generell: "Es gibt zahllose plausible Dimensionen der Variation. Hier muß man so vorgehen wie in den Wissenschaften sonst - nämlich mehr oder minder gut motivierte Hypothesen aufstellen, sie überprüfen, verfeinern, wieder überprüfen, und so fort, bis man sich allmählich an jene herantastet, die in der Tat verantwortlich sind." Daß zahllose plausible Dimensionen in Betracht gezogen werden könnten, durch die man sich hindurchvariieren kann, halte ich für einen Irrtum, den Kleins eigene Annäherung an die Textvertrautheit Lügen straft.
} 
die Relation zwischen zwei Kenntnissystemen der gleichen Personen, nämlich der Sprachkenntnis und der Kenntnis, die die Grundlage für ästhetische Urteile ist, also die Relation zwischen dem was oben Sprachkenntnis $G$ und poetische Kompetenz $P$ genannt wurde. Deren Beziehung ist aber inhärent gegeben: Poetische Eigenschaften bauen auf den Bedingungen der Sprachkenntnis auf.

Das klingt zirkulär: Daß und wie eine Person poetische Eigenschaften bewertet, wird erklärt durch die Kenntnis auf der das Verständnis poetischer Eigenschaften beruht. Tatsächlich ist diese Feststellung nicht mehr zirkulär als die unstrittige Annehme, daß das Verstehen einer sprachlichen Äußerung erklärt wird durch die Sprachkenntnis. In beiden Fällen besteht die empirisch Aufgabe darin, zu klären, was dias jeweilige Kenntnissystem enthält.

Der scheinbar besondere empirische Appeal des von Klein skizzierten Programms verlangt daher in Wahrheit sinnvolle Annahmen über die Kenntnisstrukturen, auf denen poetisch relevante Eigenschaften von Texten beruhen, nicht aber die Exploration beliebig variierbarer Merkmale von Personen wie Alter, Herkunft, Geschlecht oder Schulbildung. Die Ergebnisse geben andernfalls nicht Auskunft über die Grundlage und Natur ästhetischer Urteile, sondern über Gegebenheiten der Zeitgeschichte, des Bildungssystems, der Jugendkultur, der Migrationseffekte, oder andere Faktoren, denen die Funktion von literarischen Texten natürlich allemal auch unterliegt.

\section{2. Poetische Universalien}

Walking and jumping are innate capabilities of every human. Some of us practice these abilities to the point where others will watch them, even pay money for the privilege. In parallel fashion, all of us produce (linguistic) utterances/discourses that have aesthetic value. In part this ability must be innate: surely Shakespeare possessed an ability that none of us could hope to acquire, but lesser talents no doubt learned how to write poems or plays or novels and got better at it. Practice usually helps.

\section{Morris Halle}

Unter einem Gesichtspunkt, der nichts mit Kleins Überlegungen zu tun hat und der auch in der Skizze in Abschnitt 1 nicht vorkommt, ist es allerdings möglich, ja notwendig, Unterschiede zwischen Personen und Personengruppen im Hinblick auf poetische Kompetenz in Betracht zu ziehen. Der Vergleich von Linguistik und Poetik bzw. Sprachkenntnis und poetischer Kompetenz bringt hier einige Aspekte ans Licht, die für empirische Untersuchungen nicht nur wichtig sind, sondern ihnen erst eine ernsthafte Grundlage geben.

Ein beinahe trivialer, aber für den Charakter und die Verlässlichkeit poetischer Bewertungen folgenreicher Gesichtspunkt ist der Zusammenhang und die Differenz zwischen Sprechen und Verstehen, Sprachproduktion und Perzeption und übrigens auch Schreiben und Lesen und - damit verbunden, aber nicht identisch - aktiver und passiver Sprachbeherrschung. Daß zwischen den verschiedenen Modi der 
Sprachverwendung Unterschiede bestehen, ist jedem vertraut, Bedingungen und Ausmaß solcher Unterschiede zu bestimmen, ist allerdings weniger einfach. Klar ist indes, daß sie in Bezug auf Poesie und deren Bewertung ungleich ausgeprägter sind und damit die Variation nicht nur beim Schreiben, sondern auch bei der Rezeption und Beurteilung literarischer Texte erheblich instabiler und variantenreicher ist. In bestimmten Grenzen gilt hier, wie Morris Halle konstatiert, daß Übung hilft. Und die Bedingungen, die dabei eine Rolle spielen, können Gegenstand eines eigenen Forschungsprogramms sein, das allerdings eher didaktische Orientierung hätte. Denn für das, was eine Gedicht von Hölderlin, ein Stück von Büchner oder eine Tragödie von Sophokles bedeutend macht, ist nur sehr bedingt die pädagogische Vorbereitung der Leser von Interesse. (Eher umgekehrt wird man sich didaktisch bemühen, weil und wenn der Rang eines Textes das rechtfertigt.)

Einen anderen Aspekt, der für die Frage nach der Basis ästhetischer Bewertungen entscheidend ist, markiert die linguistische Unterscheidung zwischen Sprachfähigkeit und Sprachkenntnis, oder technisch gesprochen: der Universalgrammatik UG und der jeweils einzelsprachlichen Grammatik G. Was genau die Sprachfähigkeit festlegt, ist das Kernproblem der Linguistik, und die Antworten, die nur auf indirektem Weg gefunden werden können und entsprechend kontrovers diskutiert werden, haben sich im Lauf der letzten fünf Jahrzehnte beträchtlich gewandelt. Unstrittig gehören Operationen für die Bildung komplexer Ausdrücke aus elementaren Bestandteilen dazu, und unstrittig ist insbesondere, daß diese Operationen und elementaren Bestandteile die im Organismus verankerte Ausstattung sind, die den Erwerb der jeweiligen Muttersprache(n) möglich macht. Eine entsprechende Annahme hält nun (nicht nur) Chomsky für die poetische Fähigkeit für unzweifelhaft. So wie die jeweilige Sprachkenntnis G auf der Struktur UG der Sprachfähigkeit beruht, ist also die jeweilige poetische Kompetenz $P$ die Ausprägung einer generellen Poesiefähigkeit UP. Diese Überlegung führt zu drei möglichen Fragestellungen, die unterschiedliche, aber miteinander zusammenhängende Forschungsvorhaben bestimmen würden:

(1) Wie und durch welche Erfahrungen entsteht P auf der Basis von UP?

(2) Welche verschiedenen poetischen Systeme P gibt es aufgrund von UP?

(3) Was ist der Charakter und der Inhalt von UP?

Frage (1) betrifft die Analogie zum Spracherwerb, und man wird annehmen müssen, daß unbeschadet der Einbettung in den gleichen ontogenetischen Gesamtprozeß relevante Unterschiede zu gewärtigen sind - nicht nur wegen des Phänomens der Sonderbegabung, die stets die Basis großer Kunst ist und mit dem zusammenhängt, was nach Chomskys Überzeugung niemand erklären kann. Für den normalen Verlauf der Ontogenese von $\mathrm{P}$ gibt es jedoch reiche Evidenz und rationale Forschungsprogramme, die sich keineswegs in unbekanntem Neuland verlieren. Kinderreime und Abzählverse, spontane und interaktive Äußerungen mit Eigenschaften, die Poetische Strukturen manifestieren, bieten unmittelbar einschlägige Ansatzpunkte. Die spontane Entwicklung und ihre Begleitung ist übrigens nicht identisch mit den zuvor erwähnten Übungsprogrammen, auch wenn zwischen beidem keine strikte Trennlinie besteht.

Frage (2) führt zu mindestens zwei Problemen. Das erste setzt mit der Feststellung ein, daß es - vergleichbar mit der Verschiedenheit der Einzelsprachen unterschiedliche Poetik-Systeme gibt, die sich gleichwohl der gleichen Disposition zur poetischen Gestaltung verdanken. Auch hier geht es nicht um unbekanntes 
Terrain. Einsichten in die Formen chinesischer, arabischer, afrikanischer oder isländischer Lyrik, in die Struktur von No-Spielen und griechischen Tragödien, den Aufbau indischer oder finnischer Epen sind reiche Erfahrungsbereiche, an denen die Ermittlung von Invarianten ansetzen kann, die zu Kandidaten für die Prinzipien von UP führen können.

Das zweite Problem betrifft die Identifizierung der Variationen, die innerhalb von UP möglich sind, und damit zugleich die Rahmenbedingungen, innerhalb deren die Einzelsysteme sich ausprägen, tradiert werden, sich historisch verändern. Hier am ehesten müssten Untersuchungen der Art einsetzen, die Klein zur Bestimmung der Eigenschaften von Personen ins Auge fasst: Die Formen des Minnesangs, der Skaldendichtung, aber auch der Rap-Texte sind an strikte Rahmenbedingungen gebunden, die formal-poetische mit sozialen Strukturen verbinden.

Frage (3) kann - wie die Suche nach den Prinzipien der Sprachkenntnis - nur durch die systematische Aufklärung der in verschiedenen Systemen der Poetizität realisierten Möglichkeiten beantwortet werden. Welche Schritte damit gemeint sind, sollten die Analysen in Abschnitt 2 exemplifizieren. Auch hier gilt natürlich, daß die Frage kein unerforschtes Terrain betrifft. Eine reiche Erfahrungsgrundlage besteht für die Prinzipien von Metrik und Versbau sowie für Phänomene wie Parallelismus. Ganz andere Arten von Struktur und Regularität sind allerdings verbunden mit der unterschiedlichen Organisation von Erzählformen sowie mit Texten für dramatische Aufführungsmodalitäten.

\section{Poetik und Ästhetik}

\section{1. Parallelen und Unterschiede}

Ich habe bisher keinen systematischen Unterschied zwischen poetischen und ästhetischen Qualitäten und Bewertungen eines Textes gemacht. Und in der Tat wird man im Prinzip literarische Texte als sprachliche Gebilde mit speziellen ästhetischen Eigenschaften betrachten. Damit wird aber die Frage verdeckt, ob das Schreiben, Verstehen und Bewerten von Gedichten, Erzählungen oder Dramen in einer besondere Perfektion der Sprachausübung besteht - so wie Laufen, Springen, Schwimmen oder Schifahren bei besonderer Perfektion preiswürdig werden - oder ob die ästhetische Qualität eines Textes auf einer besonderen Kenntnisstruktur beruht, wie es in der in Abschnitt 1 resümierten Vorstellung mit der poetischen Kompetenz $\mathrm{P}$ angenommen wird. Für eine solche Kenntnisstruktur spricht unter anderem die im Zusammenhang mit der Frage (2) erwähnte mindestens partielle Lern- und Kulturabhängigkeit: Obwohl wenn man z.B. vom Charakter eines Distichons oder einer Ghasele durchaus auch ohne Anleitung etwas erfasst, bedarf eine angemessene Würdigung doch mehr als nur der Sprachkenntnis. Folgt man dieser Grundvorstellung, dann entsteht aber die Frage, ob die als Basis für die Ausbildung von $\mathrm{P}$ angenommene Fähigkeit UP Grundlage für ästhetische Strukturen und Bewertungen generell oder für solche von sprachlichen Gebilden im besonderen ist. Denn daß literarische Qualität direkt mit ästhetischer Bewertung zusammenhängt, wird intuitiv gewiß zu Recht unterstellt und entspricht der wechselnden Benennung. Wie aber verhält sich poetische Qualität generell zu ästhetischen Eigenschaften in anderen Bereichen? Zu den Fragen (1) bis (3) ergeben sich damit zwei weitere Probleme: 
(4) Wie verhält sich P und die zugrundeliegende Disposition UP zu ästhetischen Bewertungen in beliebigen anderen Domänen?

(5) Gibt es eine generelle Disposition für die Bewertung ästhetischer Qualitäten und was ist ihr Inhalt?

$\mathrm{Daß}$ es ästhetische Qualitäten in unterschiedlichen Bereichen gibt, ist offensichtlich, und auch, daß dabei Gemeinsamkeiten und wichtige Unterschiede bestehen. Ehe ich auf denkbare Folgerungen eingehe, die sich aus diesen Feststellungen für Antworten auf die Fragen (4) und (5) ergeben, will ich einen Unterschied erörtern, der das gängige Vorverständnis dieser Fragen prägt.

Für Musik und Dichtung, zwei benachbarte, zentrale Bereiche, die diese Fragestellung betrifft, weist die akademische Zuständigkeit und auch die Alltagsauffassung eine aufschlussreiche Asymmetrie auf. Beide Bereiche sind durch offenkundige Gemeinsamkeiten, aber auch durch essentielle Unterschiede geprägt. Gemeinsam ist innen vor allem der primär akustische Charakter ihrer Strukturen, die sich als Ereignisse im Zeitablauf manifestieren und damit den gleichen Realisierungsmodus aufweisen, durch den sie in den verschiedenen Formen des Gesangs auch direkt miteinander verbunden sein können. Der entscheidende Unterschied besteht darin, daß Dichtung eine konventionell-symbolisch bedingte, begriffliche Bedeutung ausdrückt, die sich essentiell von der Organisation ihrer akustisch realisierten Form unterscheidet, während die Musik eine analog-ikonische Struktur besitzt, die keine von der Signalstruktur verschiedene Bedeutung repräsentiert. Diese Bedingungen führen zu systematischen Konsequenzen verschiedener Art. Hier ist von Interesse, daß die Beschäftigung mit sprachlichen Kunstwerken in zwei Disziplinen geschieht, von denen die Literaturwissenschaft vor allem die ästhetischen Eigenschaften behandelt, während die Linguistik sich primär mit ihrer sprachliche Grundlage befasst. Musikalische Gebilde werden dagegen in einer Disziplin thematisiert, die diese Teilung nicht kennt - Musik wird im Prinzip als ästhetische Erscheinung verstanden ${ }^{5}$. Daß sprachliche Gebilde unter zwei Gesichtspunkten behandelt werden, für die in der Musikwissenschaft eine parallele Teilung nicht existiert, hat verschiedene Gründe. Der wichtigste ist zweifellos die eigenständige Struktur der Sprache, die zu trennen ist von der Vielfalt ihrer Funktionen, die sich wiederum ergeben aus dem begrifflich-symbolischen Charakter der Semantik natürlicher Sprachen. Aber welche Gründe oder Zufälligkeiten auch die disziplinäre Verfasstheit von Literatur-, Sprach- und Musikwissenschaft hervorgebracht haben, sie ist Ausdruck einer unterschiedlichen Rolle des Ästhetischen. Auch die Struktur des musikalischen Materials (in den verschiedenen Deutungen, die dieser Terminus erfahren hat), ist vornehmlich unter ästhetischen Gesichtspunkten behandelt worden, während die Sprache, falls man sie als Material der Dichtung zu betrachten hätte, ein zunächst ganz anders geartetes Gebiet darstellt.

Dieser Feststellung zufolge ist für den akademischen wie für den Alltagsverstand die Musik ein grundsätzlich ästhetisches Gebiet, während die Sprache ihre ästhetische Funktion erst als zusätzliche Möglichkeit erhält. Soweit diese Perspektive angemessen ist, ergibt sie für die Fragen (4) und (5) eine deutliche Asymmetrie in der Rolle und der Entstehung des Ästhetischen: In der Musik ist der ästhetische

\footnotetext{
${ }^{5}$ Tatsächlich ist das Bild komplizierter, da in beiden Bereichen zumindest traditionell noch die Teilung in historische und systematische Teilbereiche berücksichtigt werden muß, wie ich In Bierwisch (1979) vorgeführt habe. Für die hier anstehende Frage ist das einfache Schema durchaus angemessen.
} 
Rang offenbar direkt verbunden mit der Perfektion des Musik-Machens, dessen Funktion ja vorab auf das Ästhetische gerichtet ist, in der Dichtung hingegen tritt die ästhetische Qualität zu dem, was die Sprachkenntnis an Möglichkeiten enthält, durch die poetische Kompetenz hinzu.

Dieser eigenartige Befund entspricht einer in der Sache liegenden partiellen Asymmetrie. Einerseits unterliegen beide Bereiche in wesentlicher Hinsicht gleichen Bedingungen: So wie die Hervorbringung und Bewertung von Sprachkunst durch die poetische Kompetenz $\mathrm{P}$ bedingt und ermöglicht wird, muß für die Organisation musikalischer Strukturen eine Musikalische Kompetenz $M$ angenommen werden. Für sie gelten ähnliche Bedingungen wie die oben für $\mathrm{P}$ erläuterten: Beide sind Ausprägungen einer zugrundeliegenden Fähigkeit, beide unterliegen kulturellhistorischer Variation und Veränderung, beide sind das Ergebnis ontogenetischer Entwicklung, und beide weisen innerhalb der biologisch gegebenen Invarianz beträchtlichen individuelle Unterschiede auf.

Andererseits ergibt sich ein entscheidender Unterschied aus der Tatsache, daß die Strukturen, die durch $P$ mit einer poetischen Form versehen werden, zunächst oder parallel durch das sprachliche Kenntnissystem $G$ determiniert sind und dadurch insbesondere eine begrifflich organisierte semantische Interpretation tragen, die natürlich auch für die von $\mathrm{P}$ bedingten Eigenschaften eine zentrale Rolle spielt, eine Bedingung, für die es in den durch $\mathrm{M}$ ermöglichten musikalischen Strukturen im Prinzip keine Parallele gibt. Insofern ist die ungleiche Behandlung sprachlicher und musikalischer Gebilde ein Reflex sachlicher Gegebenheiten.

Die Parallelität der mit $P$ und $M$ abgekürzten Kenntnissysteme wirft nun allerdings eine nicht triviale Frage in Bezug auf ihre Grundlage auf: Muß für den Bereich (kulturell ausgeprägter) musikalischer Kenntnissysteme $M$ eine eigene Disposition UM angenommen werden, analog zu UP als Basis für $P$ ? Zweifellos gehört im Prinzip die Fähigkeit zum Bilden und Verstehen musikalischer Äußerungen nicht weniger zur Artausstattung als die Sprachfähigkeit und die der poetischen Gestaltung. Und es liegt auch nahe, die dafür verfügbaren Bedingungen als nicht weniger spezifisch anzusehen als die für andere mental fundierte Systeme ${ }^{6}$. Allerdings wirkt die Annahme jeweils eigener Grundlagensysteme nicht nur willkürlich und schematisch, sie verdeckt vor allem den aufzuklärenden Zusammenhang, den ja die ästhetische Orientierung und Unterscheidungsfähigkeit auch verlangt.

Es liegt daher nahe, für die in (4) und (5) gestellten Fragen eine andere Perspektive anzunehmen und die ästhetische Disposition als einen gemeinsamen Faktor zu verstehen.

\section{2. Zwei Perspektiven}

Daß ästhetische Bewertung und ästhetische Gestaltung intuitiv als einheitliches Phänomen verstanden werden, heißt nicht zwangsläufig, daß dem ein eigenes und einheitliches mentales System zugrunde liegt. Die Raumorientierung etwa wird als eigenständige Verhaltensform durch das Zusammenwirken verschiedener

\footnotetext{
${ }^{6}$ Ligeti und Neuweiler (2007), ein Komponist und ein Neurobiologe haben darüber einen ungewöhnlichen Dialog geführt, der die biologische Fähigkeit zur Steuerung der Feinmotorik als wesentliche biologische Basis nahe legt.
} 
Systemkomponenten bewirkt, visuelle, taktile, auditive Wahrnehmung, Motorik, Gleichgewichtsorgan erzeugen gemeinsam eine funktional ganzheitliche Dimension der Verhaltenorganisation mit einheitlicher Struktur. In diesem Sinn sind prinzipiell zwei Sichtweisen für das Phänomen der ästhetischen Verhaltens- und Bewertungsmöglichkeiten denkbar.

(A) Zu den Grunddispositionen des Organismus gehört die Regulation und Steuerung des Verhaltens durch ein System ästhetischer Strukturbildungen und Wertungen, die sich für verschiedene Verhaltensbereiche in entsprechenden Kenntnissystemen manifestieren.

Inhaltlich ist für ein solches System an Prinzipien zu denken, die Eigenschaften folgender Art auszeichnen:

(a) Symmetrie, Proportion

(b) Wiederholung, Kontrast

(c) Balance von Invarianz und Varianz

Sie dürften zusammenhängen mit Bedingungen der Wahrnehmungsorganisation, der Gedächtnisökonomie, der motorischen Aktivitätsregulation. Wirkungsfelder solcher Auszeichnung sind dann primäre Verhaltensbereiche wie Motorik, die verschiedenen Sinnesmodalitäten, Lautgebung, und selbstverständlich Sprache. Bezogen auf die jeweiligen Verhaltensbereiche bilden sich auf diese Weise Kenntnissysteme wie die mit $P$ und $M$ abgekürzten, denen andere an die Seite zu stellen sind. Die Gemeinsamkeiten von Musik und Tanz, von Musik und Dichtung, von Drama und Pantomime werden damit nicht erzeugt, aber strukturiert durch das angenommene ästhetische Grundpotenzial.

Die Frage (5), die auf eine ästhetischen Grunddisposition und ihren Inhalt zielt, würde damit zu verfolgen sein durch die Ermittlung und Begründung von Prinzipien wie sie unter (a) bis (c) angedeutet sind. Für die Frage (4) nach dem Verhältnis dieser ästhetischen Disposition zu Kompetenzen wie $\mathrm{P}$ und $\mathrm{M}$ würde dann die Annahme naheliegen, daß diese die jeweils domänenspezifische, ontogenetisch entstehende Ausprägung der ästhetischen Grunddisposition sind. P wäre dann nicht auf UP sondern die ontogenetische Interaktion von Spracherfahrungen mit der ästhetischen Grunddisposition zurückzuführen. Entsprechend wäre M die Ausprägung, die auditivmotorische Erfahrungen in Interaktion mit der ästhetischen Disposition ergeben.

Ich halte diese Erwägungen für durchaus plausibel und nicht bloß spekulativ. Dennoch ist klar, daß sie zunächst kaum mit einem rationalen Forschungsprogramm zu verbinden sind. Auch wenn man die in (A) umschriebene Auffassung für gültig hält, ist es daher angezeigt, eine Perspektive zu formulieren, die näher an empirische Überprüfbarkeit heranführt und sinnvoller von Resultaten ausgehen kann, die in einzelnen der in Betracht zu ziehenden Bereiche verfügbar sind:

(B) Für bestimmte Bereiche (wie Dichtung, Musik, Tanz, Malerei, Skulptur) entstehen in der Ontogenese Kenntnissysteme, die entsprechende Verhaltensleistungen und Wertungen ermöglichen. Bereichsübergreifende Eigenschaften und Prinzipien, die sich in solchen Leistungen manifestieren, sind auf entsprechende generelle ästhetische Prinzipien zurückzuführen. 
Diese Formulierung ist auf dem Hintergrund der bisherigen Erwägungen zu verstehen. Sie spart aber sowohl Dispositionen wie UP, UM und analoge Systeme wie auch die Annahme einer generellen ästhetischen Disposition aus und zielt zunächst auf die Analyse der Kenntnissysteme und die aus innen zu entnehmenden Prinzipien. Was das für die poetische Kompetenz besagt, ist in Abschnitt 2 exemplifiziert und leicht durch weitere Analysen zu ergänzen. Für die tonale Musik haben Lerdahl und Jackendoff (1983) einen großangelegten und interessanten Ansatz entwickelt. Die Prinzipien und bereichsübergreifenden oder mindestens vergleichsträchtigen Eigenschaften die sich dabei ergeben, liegen sehr wohl in dem durch die Punkte (a) bis (c) angedeuteten Bedingungen. Mit gebührender Vorsicht kann man in dieser Perspektive eine induktive Version der mit (A) umschriebenen Vorstellung sehen. Das Programm, das sich damit abzeichnet, setzt durchaus auf empirisches Vorgehen - wenn auch nicht auf einfache Tests und induktive Verallgemeinerung von Beobachtungsdaten, aber doch auf ein Vorgehen, wie Klein es zusammengefaßt hat (Anmerkung 4), "nämlich mehr oder minder gut motivierte Hypothesen aufstellen, sie überprüfen, verfeinern, wieder überprüfen, und so fort, bis man sich allmählich an jene herantastet, die in der Tat verantwortlich sind."

Wenn dieser Ansatz erfolgreich ist, wird er schrittweise erklären, wie ästhetische Urteile über Gedichte entstehen, auf welchen Eigenschaften sie beruhen, welche Prinzipien innen zugrunde liegen. Vielleicht ist aus einfachen Prinzipien abzuleiten, was ein großes Gedicht ist.

Was an all dem fasziniert, wird dennoch die Aura behalten, in der sich das manifestiert, was keiner erklären kann.

\section{Literatur}

Bierwisch, Manfred (1965) Linguistik und Poetik, in Helmut Kreuzer und Rul Gunzenhäuser S. 49-65

Bierwisch, Manfred (1979) Musik und Sprache: Überlegungen zu ihrer Struktur und Funktionsweise, in Peters Jahrbuch 1978, S. 9-102 Leipzig: Edition Peters

Fieled, Adam (2008) Mini-Interview mit Noam Chomsky und Morris Halle, Internet

Klein, Wolfgang (2007) Wie ist eine exakte Wissenschaft von der Literatur möglich, Zeitschrift für Literaturwissenschaft und Linguistik

Kreuzer, Helmut und Rul Gunzenhäuser, (1965) Mathematik und Dichtung, München

Lerdahl, Fred and Ray Jackendoff (1983) A Generativ Theory of Tonal Music, Cambridge, Mass.

Ligeti, György und Gerhard Neuweiler (2007) Motorische Intelligenz, Berlin

Taubes, Jacob (1991) Abendländische Eschatologie, München 